\title{
Psychological Impact of Ambiguous Health Messages about COVID-19
}

\author{
Nicolle Simonovic $^{1}\left[\right.$ Jennifer M. Taber ${ }^{1}[$
}

Received: 7 May 2021 / Accepted: 4 November 2021 / Published online: 23 November 2021

(C) The Author(s), under exclusive licence to Springer Science+Business Media, LLC, part of Springer Nature 2021

\begin{abstract}
Perceiving ambiguity in health informationthat is, uncertainty elicited from believing information lacks credibility, reliability, or adequacy-is typically associated with pessimistic appraisals (e.g., high perceived disease risk) and behavioral avoidance. We examined the effect of ambiguous health information about COVID-19 on health cognitions and vaccination intentions, and tested a "normalized-uncertainty" intervention. Two studies with identical methodology (online adult sample: $n=299$, undergraduate sample: $n=150$ ) were conducted in March to April 2020. Participants were randomly assigned to read one of three health messages about COVID-19 that emphasized what was currently unknown (ambiguity condition), what was currently unknown but that scientific uncertainty is expected (intervention condition), or what was currently known (control condition). The ambiguity condition led to greater perceived ambiguity than the control condition and perceived ambiguity in the intervention condition was comparable to the ambiguity condition. There were few differences in health cognitions, and no differences in vaccination intentions, when examining pairwise comparisons across the three conditions. Correlational analyses collapsing across condition indicated evidence of pessimistic appraisal but not behavioral avoidance among individuals who perceived greater ambiguity. Future research should examine longer, more detailed normalized-uncertainty interventions.
\end{abstract}

Keywords Ambiguity $\cdot$ Risk perceptions $\cdot$ Health behavior

Nicolle Simonovic

nsimonov@kent.edu

1 Department of Psychological Sciences, Kent State University, 390 Kent Hall, Kent, OH 44242-0001, USA

\section{Introduction}

In medical contexts, scientists and experts often do not have all the answers or definitive information about the best course of action. This is particularly true with novel or unprecedented health treatments, diseases, or devices, such as electronic cigarettes (Katz et al., 2017, 2018; Pepper et al., 2019; Simonovic \& Taber, 2020) or lung cancer screening (i.e., low-dose computed tomography; Schapira et al., 2016; Yang et al., 2019). SARS-CoV-2 (more colloquially referred to as COVID-19 or the novel coronavirus) is a global pandemic that emerged in the United States in January 2020 about which very little scientific information was known with certainty at the start of the pandemic (CDC, 2020). In March 2020, when COVID-19 was first considered a worldwide pandemic (WHO, 2020a), experts did not have precise understanding of the extent and nature of the health risks or how best to prevent transmission. As a result, medical authorities provided conflicting recommendations to the lay public, such as about whether to wear a mask (see Liang et al., 2020 for an overview of the contradicting perspectives as well as robust evidence that face masks reduce transmission). However, the extent of uncertainty about COVID-19 reflects the nature of scientific inquiry: advances are made continuously, and information accumulates through ongoing research.

Information emerging from ongoing research can lead people to experience and perceive ambiguity. According to a conceptual taxonomy of uncertainty in health care, ambiguity is a type of uncertainty (Han et al., 2011a, 2011b) that people experience when information is lacking in reliability, credibility, or is in some way inadequate (Camerer \& Weber, 1992; Ellsberg, 1961). Ambiguity can be thought of as uncertainty that arises from limitations in the existing knowledge base about a particular topic. Some people who 
perceive greater ambiguity about a particular topic engage in pessimistic appraisal and behavioral avoidance; these responses to ambiguity have been termed ambiguity aversion as a general pattern of responding. The term ambiguity aversion is also used to describe an extreme in a continuum of individual level differences in tolerance for ambiguity, such that individuals high in ambiguity aversion are said to have low tolerance for ambiguity (see Simonovic et al., 2020 for a discussion of the different uses of the term "ambiguity aversion"). Pessimistic appraisal in health contexts has been shown as associations of greater perceived ambiguity with greater worry (Han et al., 2006, 2007a, 2007b), decreased trust in health officials and lower reliance on doctors (Han et al., 2018; Simonovic et al., 2020), and lower perceived response and self-efficacy (Simonovic et al., 2020; Taber et al., 2015). All of these constructs are health cognitions that are often associated with lower engagement in preventive behavior. Behavioral avoidance, or avoiding options that have unknown probabilities, has been shown as lower intentions to engage in a behavior [e.g., hesitancy to vaccinate (Blaisdell et al., 2016; Han et al., 2018; Meszaros et al., 1996; Ritov \& Baron, 1990)]. These findings have important consequences: for example, a person who perceives higher ambiguity about COVID-19 might also be more likely to express behavioral avoidance through hesitancy to vaccinate, thus increasing their risk of contracting COVID-19 and potentially spreading it to other people.

A strategy that might promote adaptive responses to ambiguous information is normalization of uncertainty (Han et al., 2018). According to the "Competence Hypothesis," people respond aversively to ambiguity when they perceive themselves as having low competence-that is, if they do not feel knowledgeable-in a particular area of decision-making (Han et al., 2018; Heath \& Tversky, 1991; Klein et al., 2010). Making people feel more competent in assessing their risk, such as by emphasizing that risk is unknowable for everyone rather than for a specific individual, can decrease the maladaptive effects of ambiguous information (Chow \& Sarin, 2001, 2002). Based on these findings, Han et al. (2018) hypothesized that emphasizing that uncertainty is common and thus expected-a strategy they termed "normalized-uncertainty"-might promote more adaptive responses to ambiguous information within the context of a hypothetical health pandemic which was described as preventable by a vaccine. An important distinction about normalization of uncertainty is that it should not decrease ambiguity, but rather influence how people respond to ambiguity. In an online study about a hypothetical health pandemic, Spanish adults were assigned to read an ambiguous health message, an unambiguous health message, or an ambiguous health message coupled with text suggesting that uncertainty is normal and expected (Han et al., 2018). Contrary to hypotheses, normalization of uncertainty did not decrease maladaptive health cognitions associated with ambiguity. The low external validity regarding the hypothetical health pandemic may have undermined the efficacy of this strategy, such that normalization of uncertainty during an actual health pandemic might be more effective. More specifically, a non-hypothetical situation should increase relevance and thus engagement with the message, such that a normalized-uncertainty message about a real pandemic may be more likely to elicit responses consistent with how people would respond if they came across ambiguous information in their day-to-day lives than would a message about a hypothetical scenario. Thus, a real-world context may provide more insight into intervention efficacy. Because a real-world contextmay provide more insight into intervention efficacy, (DeAngelis, 2021; Salive, 2017), the aim of the present study was to examine whether a normalization of uncertainty intervention promoted adaptive responses to ambiguous health information about COVID-19.

Although the normalization of uncertainty intervention did not work in this prior study, results demonstrated several maladaptive effects of ambiguous information on health cognitions (Han et al., 2018). Specifically, participants who read a message emphasizing ambiguity about a vaccine for the hypothetical disease reported less trust in public health officials, lower perceived vaccine effectiveness, and most importantly, lower intentions to vaccinate than participants who read a control message in which ambiguity was not emphasized (Han et al., 2018). The finding that the ambiguity condition led to lower intentions to vaccinate is consistent with the behavioral avoidance typically associated with ambiguity aversion. Researchers also demonstrated a surprising moderating role of health literacy, such that individuals who were more health literate reported lower intentions to vaccinate when exposed to ambiguous health information compared to participants who read a control message (i.e., participants who were more health literate were more ambiguity averse). Ambiguity aversion is considered to be a "highly moderated phenomenon" (Han et al., 2011b, p. 361 ) and so it is important to further examine the role of health literacy and other moderators in research on ambiguity. Further, participants who read ambiguous health information also perceived lower likelihood of and lower severity of contracting the disease at the center of the hypothetical health pandemic, which is inconsistent with a pattern of pessimistic appraisal. Perceived severity and likelihood (also called susceptibility) are two distinct dimensions of risk perceptions (El-Toukhy, 2015). Risk perceptions, or subjective beliefs about one's risk, are an important predictor of health behavior (Brewer et al., 2007; Ferrer \& Klein, 2015). As part of subjective beliefs about risk, susceptibility perceptions refer to one's chance of experiencing a negative outcome, 
whereas severity perceptions refer to people's expectations of how serious or harmful the negative outcome would be (Brewer et al., 2007; El-Toukhy, 2015).

\section{Hypotheses}

Here, we report the results of two experiments that were conceptualized as direct replications. Hypotheses were the same for both experiments. First, we hypothesized that participants randomly assigned to read an ambiguous message about COVID-19 (ambiguity condition) would report more maladaptive health cognitions (i.e., lower risk perceptions, self-efficacy, and trust in public health officials and doctors) and lower intentions to vaccinate compared to participants who received a normalization of uncertainty intervention (intervention condition) and to participants who read an unambiguous message (control condition). This hypothesis was based in part on prior research in which perceived ambiguity was associated with lower perceived self-efficacy (Simonovic et al., 2020), and lower trust in health officials (Han et al., 2018). Although hypothesizing that ambiguity would lead to lower risk perceptions is inconsistent with theoretical background (i.e., pessimistic appraisal), this hypothesis was based on the effect of ambiguity on risk perceptions in the study informing the current study (Han et al., 2018). Further, whether lower or higher risk perceptions are maladaptive is context-dependent, and we expected that lower disease risk perceptions would be maladaptive in the present context (i.e., if people underestimate their risk, they might be less likely to engage in health protective behavior).

Second, we hypothesized that among participants who read an ambiguous message about COVID-19, those with higher, dispositional optimism, and tolerance for ambiguity would report more adaptive health cognitions. We included health literacy because it was a significant moderator of effects in the study which informed the present one (Han et al., 2018), but because the direction of their effect was opposite of predictions, we did not have a directional hypothesis for health literacy. We hypothesized moderating effects of dispositional optimism because of prior research suggesting that individuals with greater optimism (Biesecker et al., 2014; Han et al., 2011b; Taber et al., 2015) would respond more positively to ambiguous and uncertain health information. Finally, we also examined moderating effects of tolerance for ambiguity, as this variable can influence responses to ambiguous health information (Han et al., 2014). To reduce the number of analyses and the likelihood of Type I error, we only conducted moderation analyses on our primary dependent variables of interest: perceived susceptibility, worry, severity (all conceptualized as aspects of risk perceptions), and behavioral intentions. These variables were of interest because they are commonly examined in research on ambiguity in health contexts (e.g., Han et al., 2006, 2007a, 2007b, 2018; Pepper et al., 2019).

\section{General method}

\section{Overview}

Two experiments were conducted. The online sample recruited for Experiment 1 was older, $M=56.36, S D=16.41$, range $=18-91$ years, than the undergraduate sample recruited for Experiment 2, $M=19.93, S D=1.81$, range $=18$ to 29 years. A CDC report indicated less uptake of COVID19 protective behaviors among younger adults than older adults (Hutchins et al., 2020), thus supporting our decision to collect separate data from a student sample and a sample of online adults. Kent State University's IRB approved both experiments. Criteria for eligibility included aged 18 years and older, fluency in English, and no self-reported prior or current diagnosis of COVID-19. Data from both experiments were collected between March to April 2020, shortly after COVID-19 was first considered a worldwide pandemic on March 11th (WHO, 2020a) and lockdowns were first implemented across the U.S. (Moreland et al., 2020). Of note, data collection from both experiments occurred simultaneously, and although we consider both experiments to be direct replications of each other, we report data from Experiment 1 first simply because data collection was completed first and because we considered these data to be a stronger test of our research questions (as the sample in Experiment 1 was adequately powered, whereas the sample in Experiment 2 was somewhat underpowered, as explained in the Methods). The procedure was consistent across studies and measures were nearly identical (except for education). Sample sizes were determined from an a priori power analysis conducted in $G^{*}$ Power 3.1.9.2 (Faul et al., 2007). According to the power analysis, a sample size of 159 was required to conduct a univariate analysis of variance (ANOVA) with main effects and interactions with three groups (representing each of the three experimental conditions) considering 0.80 power, alpha $=0.05$, and a medium effect size. We powered for a medium effect size based on odds ratios of 1.46, 1.53, and 2.60 reported in two prior studies for the relationship between perceived ambiguity and perceived cancer susceptibility and worry (Han et al., 2006, 2007a). Because these odds ratios ranged from small-medium to large effect sizes, we powered for a medium effect size.

\section{Study design and procedure}

Both experiments, administered through Qualtrics, were described as a study about COVID-19 attitudes and beliefs. Participants who provided informed consent were randomly 
assigned to receive one of three health messages: one-third received information emphasizing what was known about COVID-19 (unambiguous control condition); one-third received information emphasizing limitations of the scientific knowledge about COVID-19 (ambiguity condition); and one-third received information emphasizing limitations of the scientific knowledge about COVID-19 and additional information to normalize the inherent uncertainty (intervention condition). After reading the health message, participants completed two items that served as a manipulation check, followed by a measure of discrete emotions (discrete emotion data are not reported here as they were considered pilot data for a future study). Next participants completed a 4-item measure of perceived ambiguity about COVID-19 used as another manipulation check followed by a questionnaire assessing health cognitions, individual differences hypothesized as moderators, and standard demographic items. Upon completing the study, participants were debriefed and given links to resources to learn more about COVID-19.

\section{Intervention}

The full text and a longer description of each health message is available on Open Science Framework (OSF; https://osf. io/n9f7x/). The three health messages were factually accurate and created using information from authoritative sources (e.g., the World Health Organization) and popular and credible media sources (i.e., the World Economic Forum). Importantly, all the information presented was considered accurate at the time of data collection (March 26, 2020).

The health messages all contained standard information about COVID-19, including a description and symptoms. After this introductory paragraph, the message differed based on condition. In the unambiguous health message, participants read about what scientists know about COVID19. In the ambiguous health message, participants read about what scientists do not know about COVID-19. Lastly, in the normalization of uncertainty message, participants read the same text from the ambiguous health message with the addition of four sentences that served to normalize uncertainty and were taken verbatim from a prior study (Han et al., 2018): "Often at the beginning of outbreaks, we just don't have all the information we wish we had. For now, we have to do the best we can with the information we do have. In life, we never have perfect knowledge of any health risks, and our information commonly changes. All we can ever do is take action based on the limited knowledge we do have."

\section{Measures}

A document including the full text of all survey measures can be found on OSF (https://osf.io/n9f7x/). Not all included measures were analyzed as part of the present study as they were not central to hypotheses. Further, we included measures of response efficacy and reactance consistent with the rationale specified in other studies (Simonovic \& Taber, 2021; Taber et al., 2015); however, after conducting analyses that demonstrated null effects, we realized that the health messages did not adequately target these variables (i.e., the messages did not make recommended actions salient or explicitly tell participants how to act). Therefore, we do not describe these analyses further, but they are available upon request from the corresponding author. Measures below are described in the order they were administered.

\section{Manipulation checks}

Two items were used as a manipulation check to assess whether perceived ambiguity differed across conditions. In the first item [adapted from Jensen et al. (2017)], participants indicated their agreement with: "There are many limitations of the existing information about COVID-19" on a scale from 1 (strongly disagree) to 4 (strongly agree). In the second item (written for this study), participants were given the same response options to indicate the extent of their agreement with: "There is a lot that is unknown about COVID-19." In Experiment 1, both items were significantly correlated and thus averaged $(r=0.61, p<0.001)$. In Experiment 2 , the associations were weaker $(r=0.39, p<0.001)$, but we averaged the items for ease of interpretation. Results did not differ when analyses were conducted with the individual items.

We also included an additional 4-item measure of perceived ambiguity about COVID-19 [adapted from Simonovic and Taber (2020) and written for the present study]. These items were introduced with, "The next set of questions will ask about your beliefs and attitudes towards the novel coronavirus, or COVID-19. These questions will refer to 'COVID-19', which we use to refer to the novel coronavirus." Participants then reported the extent to which they agreed that "leading scientists and experts...do not know enough about COVID-19/have conflicting opinions about how severe COVID-19 is and how it is spread/do not know whether the spread of COVID-19 will decrease when weather becomes warmer," and that "Whether one is at risk for COVID-19 cannot be accurately predicted or precisely measured." Items were assessed on a scale from 1 (strongly disagree) to 4 (strongly agree) and averaged such that higher values indicated greater perceived ambiguity $\left(\alpha_{E x p 1}=0.70\right.$, $\alpha_{\text {Exp } 2}=0.58$ ).

To distinguish these two perceived ambiguity measures, the former 2-item manipulation check is hereafter referred to as "perceived message ambiguity" and the latter 4-item manipulation check is hereafter referred to as "perceived COVID-19 ambiguity." We refer to the former 
2-item manipulation check as "perceived message ambiguity" because these items were broad and meant to capture the overall message of the ambiguous health message and because they occurred immediately after the messages. We refer to the latter 4-item measure as "perceived COVID-19 ambiguity" because these items were more tailored to details specific to the health context of COVID-19 and were separated from the messages by a discrete emotion scale. When referencing both, we use the term "perceived ambiguity." We retained each measure as separate scales because analyses showed some differences in zero-order correlations of each measure with outcomes.

\section{Dependent variables}

Risk perceptions were conceptualized as including perceived susceptibility of getting COVID-19, worry about COVID19 , and perceived severity of COVID-19. Susceptibility and worry were assessed with separate items referring to the self and to family members/friends.

Perceived COVID-19 susceptibility [adapted from De Zwart et al. (2009) and Klein and Ferrer (2018)] was assessed with six items, such as, "Overall, how likely is it that you will be infected with COVID-19 in the next year?" Response options varied across items but ranged from 1 to 5 and higher scores indicated greater susceptibility. Because reliability was high $\left(\alpha_{\operatorname{Exp} 1}=0.89, \alpha_{\operatorname{Exp} 2}=0.78\right)$, all six items were averaged to create a score of perceived susceptibility. "Don't know" was also provided as an explicit response option. Participants who selected "don't know" were excluded from analyses involving perceived susceptibility. COVID-19 worry [adapted from Weinstein et al. (2007) and Taber et al. (2019)] was assessed as the average of six items assessing worry, concern, and anxiety about being infected with COVID-19 $(1=$ not at all, $4=a$ lot; $\alpha_{\text {Exp1 }}=0.94, \alpha_{\text {Exp2 }}=0.91$ ).

Trust in public health officials [adapted from Eisenman et al. (2012) and Han et al. (2018)] was assessed with four items $(1=$ not at all, $4=a$ lot $)$. For example, participants were asked the extent to which they were confident that public health officials could "respond effectively to protect the health of the public." Items were averaged to create a scale $\left(\alpha_{E x p 1}=0.81, \alpha_{E x p 2}=0.80\right)$. Trust in doctors was assessed with one item adapted from the Health Information National Trends Survey (National Cancer Institute, 2003): "In general, how much would you trust information about COVID19 from a doctor?" $(1=$ not at all, $4=$ a lot $)$.

Self-efficacy [adapted from De Zwart et al. (2009)] was assessed as the average of three items $\left(\alpha_{E x p 1}=0.92\right.$, $\left.\alpha_{E x p 2}=0.92\right)$ : "How [confident/sure/certain] are you that you could prevent being infected with COVID-19?" on a scale from 1 (not at all) to 4 (very).
Perceived COVID-19 severity [adapted from Lipkus et al. (2003)] was assessed as the average of three items $\left(\alpha_{E x p 1}=0.89, \alpha_{E x p 2}=0.80\right)$ to which participants rated their agreement that COVID-19 is a serious condition, dangerous, and life-threatening on a scale from 1 (strongly disagree) to 4 (strongly agree).

Intentions to vaccinate [adapted from Han et al. (2018)] was assessed with one item on a scale from 1 (strongly disagree) to 4 (strongly agree): "At this time, there is no approved vaccine to protect against COVID-19. However, scientists are working to develop a vaccine. Please rate how much you agree or disagree with the following statement: Once a vaccine is available, I intend to get vaccinated against COVID-19."

\section{Moderators}

Tolerance for ambiguity was assessed as the average of six items $\left[\alpha_{E x p 1}=0.82, \alpha_{E x p 2}=0.72\right.$ (Han et al., 2009, 2014)]. Participants were asked to "Please imagine that you are considering having a medical test that checks for cancer. Experts have conflicting opinions about this medical test." and then rated their agreement with statements such as, "I would be afraid of trying the test" $(1=$ strongly disagree, $4=$ strongly agree). After reverse scoring necessary items, greater scores indicated higher tolerance for ambiguity. Dispositional optimism was assessed as the average of the six items $\left(\alpha_{\text {Exp } 1}=0.84, \alpha_{\text {Exp } 2}=0.82\right)$ from the Life Orientation TestRevised (Scheier et al., 1994), such as "In uncertain times, I usually expect the best" ( $1=$ strongly disagree, $5=$ strongly agree). Health literacy (Chew et al., 2008) was assessed as the average of three items $\left(\alpha_{E x p 1}=0.71, \alpha_{E x p 2}=0.53\right)$, such as about how confident participants are in filling out forms by themselves ( $1=$ none of the time, $5=$ all of the time $)$.

Finally, participants reported standard demographic factors including age in years (continuous), gender (coded as $0=$ "female," $1=$ "male"), and race (coded as $0=$ "nonwhite," 1 = "white"). Participants also reported their level of education in Experiment 1 or their year in school in Experiment 2.

\section{Pre-registration}

A brief pre-registration is available on OSF (https://osf.io/ $\mathrm{n} 9 \mathrm{f} 7 \mathrm{x} /$ ) that includes the general research question, study design, hypotheses, and power analyses and planned samples. There are differences between the preregistered plan and what is reported here. In the current paper, we clarified hypotheses to better explicate expectations for specific conditions. The current paper includes intentions to vaccinate as an outcome (not mentioned in pre-registration) but not 
perceived social support (we did not run any analyses with this variable). The preregistration only specified that we would conduct individual ANOVAs across the three groups on each dependent variable and did not include any information about testing moderators, examining bivariate correlations, or examining pairwise comparisons.

\section{Overview of analyses}

First, we used ANOVAs to test whether the manipulation led to greater perceived ambiguity in the ambiguity versus the control condition. Next, we tested whether random assignment was successful by using ANOVAs and chi-square tests to examine whether demographic factors or hypothesized moderators differed across conditions. We then examined bivariate correlations among all conceptual study variables (i.e., excluding demographics).

To test for differences in what we conceptualized as the primary (i.e., perceived susceptibility, worry, severity, and vaccination intentions) and secondary (i.e., self-efficacy, trust in officials, and trust in doctors) dependent variables, we conducted ANOVAs to test whether there were omnibus differences across the three conditions and to examine pairwise comparisons across conditions. We also tested the interactive effects of three potential moderators (i.e., tolerance for ambiguity, optimism, and health literacy) with condition on the four primary dependent variables. This was tested using regression analyses with dummy variables created for the three conditions. The continuous moderators were mean centered before creating interaction terms. Consistent with the hypotheses outlined in the pre-registration, we were interested in interactions involving the ambiguity versus control conditions (Dummy code 1) and ambiguity versus intervention conditions (Dummy code 2 ).

\section{Experiment 1}

\section{Participants}

Participants $(n=299 ; 53.8 \%$ female; $76.6 \%$ white; $M_{\text {age }}=56.36$; education: $5 \%$ high school or less, $28.8 \%$ high school graduate; $26.8 \%$ some college or associated degree completed; $30.1 \%$ college degree; $9.4 \%$ post graduate or professional degree) were U.S. adults who were recruited through Dynata and were each compensated with $\$ 5$ for the approximately 28-min study. Dynata is a research firm that recruits participants through a US-based online panel vetted from invitations sent out to thousands of organizations (e.g., award programs and social media). Participants completed the study between March 27 and April 2, 2020. Data were excluded from participants who completed the study in less than $30 \%$ of the bid length of the study $(n=42)$, failed at least one of two attention checks $(n=12)$, completed less than $60 \%$ of the survey $(n=2)$, wrote in nonsense responses to open-ended questions $(n=15)$, and had duplicate IP addresses $(n=6)$. These exclusions were not included in the final sample size noted above. The sample size drops to 263 participants for analyses including perceived susceptibility because of participants who responded to this item with the response option of "don't know."

\section{Results and discussion}

\section{Manipulation checks}

As expected (see Table 1), participants in the ambiguity condition reported significantly greater perceived ambiguity, $M=3.26$, than participants in the control condition, $M=2.92, p<0.001$. However, perceived message ambiguity

Table 1 Experiment 1: unadjusted means and standard deviations of study variables across conditions

\begin{tabular}{|c|c|c|c|c|c|c|}
\hline & \multicolumn{6}{|l|}{ ANOVAs } \\
\hline & $\begin{array}{l}\text { Control, } \\
\mathrm{M}(\mathrm{SD}) n=100\end{array}$ & $\begin{array}{l}\text { Ambiguity, } \\
\text { M (SD) } n=107\end{array}$ & $\begin{array}{l}\text { Intervention, } \\
\mathrm{M}(\mathrm{SD}) n=92\end{array}$ & $F$ & $\begin{array}{l}p \text { Value, test of } \\
\text { between subjects }\end{array}$ & $\begin{array}{l}\text { Partial eta } \\
\text { squared }\end{array}$ \\
\hline Message ambiguity & $2.92(0.75)^{\mathrm{a}}$ & $3.26(0.66)^{\mathrm{b}}$ & $3.28(0.60)^{\mathrm{b}}$ & 9.07 & $<0.001$ & 0.058 \\
\hline COVID-19 ambiguity & $2.78(0.60)^{\mathrm{a}}$ & $3.05(0.57)^{\mathrm{b}}$ & $3.19(0.54)^{\mathrm{b}}$ & 12.79 & $<0.001$ & 0.080 \\
\hline Susceptibility & $2.61(0.81)$ & $2.63(0.87)$ & $2.70(0.85)$ & 0.26 & 0.773 & 0.002 \\
\hline Worry & $2.53(0.87)$ & $2.48(0.79)$ & $2.40(0.77)$ & 0.67 & 0.512 & 0.005 \\
\hline Severity & $3.68(0.51)^{\mathrm{a}}$ & $3.50(0.64)^{b}$ & $3.69(0.50)^{\mathrm{a}}$ & 4.08 & 0.018 & 0.027 \\
\hline Vaccination intentions & $3.21(0.97)$ & $3.20(0.96)$ & $3.27(1.01)$ & 0.16 & 0.849 & 0.001 \\
\hline Self-efficacy & $2.43(0.83)^{\mathrm{a}}$ & $2.29(0.81)^{\mathrm{ab}}$ & $2.19(0.89)^{\mathrm{b}}$ & 2.09 & 0.126 & 0.014 \\
\hline Trust in public health officials & $2.76(0.71)^{\mathrm{a}}$ & $2.60(0.73)^{\mathrm{ab}}$ & $2.54(0.71)^{\mathrm{b}}$ & 2.40 & 0.092 & 0.016 \\
\hline Trust in doctors & $3.04(0.82)$ & $2.99(0.78)$ & $2.92(0.82)$ & 0.50 & 0.606 & 0.003 \\
\hline
\end{tabular}

Mean values with different superscripts differ significantly at $p<0.05$ and were drawn from the ANOVA. Absence of superscripts denotes that no pairwise comparisons were statistically significant. Degrees of freedom is 2, 260 for susceptibility and 2, 296 for all other measures 
Table 2 Experiment 2: unadjusted means and standard deviations of study variables across conditions

\begin{tabular}{|c|c|c|c|c|c|c|}
\hline & \multicolumn{6}{|l|}{ ANOVAs } \\
\hline & $\begin{array}{l}\text { Control, } \\
\mathrm{M}(\mathrm{SD}) n=49\end{array}$ & $\begin{array}{l}\text { Ambiguity, } \\
\mathrm{M}(\mathrm{SD}) n=50\end{array}$ & $\begin{array}{l}\text { Intervention, } \\
\mathrm{M}(\mathrm{SD}) n=51\end{array}$ & $F$ & $\begin{array}{l}p \text { value, test of } \\
\text { between subjects }\end{array}$ & $\begin{array}{l}\text { Partial eta } \\
\text { squared }\end{array}$ \\
\hline Message ambiguity & $2.80(0.64)^{\mathrm{a}}$ & $3.35(0.48)^{\mathrm{b}}$ & $3.27(0.47)^{\mathrm{b}}$ & 15.30 & $<0.001$ & 0.172 \\
\hline COVID-19 ambiguity & $2.49(0.56)^{\mathrm{a}}$ & $3.06(0.58)^{\mathrm{b}}$ & $2.94(0.45)^{\mathrm{b}}$ & 15.87 & $<0.001$ & 0.178 \\
\hline Susceptibility & $2.85(0.64)^{\mathrm{ab}}$ & $3.10(0.69)^{\mathrm{a}}$ & $2.82(0.70)^{\mathrm{b}}$ & 2.49 & 0.086 & 0.033 \\
\hline Worry & $2.62(0.70)$ & $2.70(0.74)$ & $2.61(0.69)$ & 0.24 & 0.786 & 0.003 \\
\hline Severity & $3.52(0.50)$ & $3.38(0.60)$ & $3.55(0.48)$ & 1.48 & 0.230 & 0.020 \\
\hline Vaccination intentions & $3.31(0.77)$ & $3.30(0.84)$ & $3.08(1.04)$ & 1.07 & 0.345 & 0.014 \\
\hline Self-efficacy & $2.19(0.75)$ & $2.27(0.75)$ & $2.35(0.96)$ & 0.45 & 0.640 & 0.006 \\
\hline Trust in public health officials & $2.57(0.63)$ & $2.45(0.76)$ & $2.56(0.72)$ & 0.42 & 0.658 & 0.006 \\
\hline Trust in doctors & $3.02(0.75)$ & $3.10(0.65)$ & $3.02(0.76)$ & 0.21 & 0.815 & 0.003 \\
\hline
\end{tabular}

Mean values with different superscripts differ significantly at $p<0.05$ and were drawn from the ANOVA. Absence of superscripts denotes that no pairwise comparisons were statistically significant. Degrees of freedom is 2, 144 for susceptibility and 2, 147 for all other measures

did not differ between the intervention and ambiguity conditions, $p=0.883$, and those in the intervention condition, $M=3.28$, reported greater perceived message ambiguity than those in the control condition, $p<0.001$. Thus, the normalized uncertainty intervention did not reduce perceived ambiguity. The same pattern of effects was observed for perceived ambiguity about COVID-19 (Table 1), providing further evidence of the successful induction of perceived ambiguity in the ambiguity compared to the control condition.

\section{Differences in demographic factors and moderators by condition}

Confirming successful randomization, there were no significant differences in age, gender, race, education, tolerance for ambiguity, optimism, or health literacy (all $p$ 's $>0.159$ ) across conditions.

\section{Associations among variables}

Table 3 provides descriptive statistics and correlations among study variables. When collapsing across conditions, greater perceived message ambiguity was significantly associated with greater perceived COVID-19 ambiguity. Individuals who reported greater perceived ambiguity also reported greater perceived susceptibility, greater worry about COVID-19, and lower perceived self-efficacy to prevent COVID-19, consistent with a pattern of pessimistic appraisal; these patterns were shown for both indices of perceived ambiguity. Those who reported greater perceived ambiguity in response to the health message also reported greater perceived COVID-19 severity, and those who reported greater perceived ambiguity about COVID-19 more generally reported less trust in public health officials and doctors. Neither measure of perceived ambiguity was significantly associated with vaccination intentions.

\section{Main effects of condition}

As shown in Table 1, a main effect of condition was found for perceived severity only; the nature of this effect was consistent with predictions of more maladaptive health beliefs - but not with pessimistic appraisals - such that the participants in the ambiguity condition reported lower perceived severity, $M=3.50$, than participants in the control, $M=3.68$, and intervention, $M=3.69$, conditions.

\section{Moderators}

We next used hierarchical linear regressions with dummy codes to test whether condition interacted with each of three possible moderators (i.e., tolerance for ambiguity, optimism, and health literacy) to predict the primary outcomes of perceived susceptibility, worry, severity, and vaccination intentions (in total, 12 regression analyses were run). Only one interaction reached statistical significance of $p<0.05$. Specifically, tolerance for ambiguity interacted with condition (Dummy 1) to predict vaccination intentions. However, because this effect would not survive correction for multiple tests and was not replicated in Experiment 2, we do not describe this interaction further.

\section{Experiment 2}

\section{Participants}

Participants ( $N=150 ; 76 \%$ female; $77.3 \%$ white; $M_{\text {age }}=19.93$; year in school: $46 \%$ freshman, $22.7 \%$ sophomore; $14.7 \%$ junior, $16.7 \%$ senior) were undergraduates who were recruited through a psychology participant pool and compensated with course credit for the approximately 27 min study (calculated 
Table 3 Correlations among study variables. Experiment 1 (online adult sample, $n=299$ ) above the diagonal, Experiment 2 (undergraduate sample, $n=150$ ) below the diagonal

\begin{tabular}{|c|c|c|c|c|c|c|c|c|c|c|c|c|}
\hline & 1 & 2 & 3 & 4 & 5 & 6 & 7 & 8 & 9 & 10 & 11 & 12 \\
\hline \multicolumn{13}{|l|}{ Manipulation checks } \\
\hline 1. Message ambiguity & - & $0.39^{* * *}$ & $0.13^{*}$ & $0.18^{* *}$ & $0.23^{* *}$ & 0.09 & $-0.16^{* *}$ & -0.03 & 0.02 & -0.04 & -0.02 & -0.02 \\
\hline 2. COVID-19 ambiguity & $0.50^{* *}$ & - & $0.21^{* *}$ & $0.16^{* *}$ & 0.09 & -0.04 & $-0.20^{* *}$ & $-0.23^{* *}$ & $-0.20^{* *}$ & -0.06 & -0.07 & 0.07 \\
\hline \multicolumn{13}{|l|}{ Dependent variables } \\
\hline 3. Susceptibility & 0.16 & $0.22^{* *}$ & - & $0.58^{* *}$ & $0.23^{* *}$ & $0.22^{* *}$ & $-0.52^{* *}$ & -0.06 & 0.04 & -0.04 & $-0.23^{* *}$ & -0.11 \\
\hline 4. Worry & 0.05 & $0.17^{*}$ & $0.43^{* *}$ & - & $0.38^{* *}$ & $0.15^{*}$ & $-0.17^{* *}$ & -0.01 & 0.04 & $-0.17^{* *}$ & $-0.29^{* *}$ & $-0.23^{* *}$ \\
\hline 5. Severity & -0.05 & 0.03 & 0.04 & $0.29^{* *}$ & - & $0.36^{* *}$ & $-0.13^{*}$ & $0.22^{* *}$ & $0.28^{* *}$ & $0.20^{* *}$ & 0.06 & $0.15^{*}$ \\
\hline 6. Vaccination intentions & 0.08 & 0.02 & 0.09 & 0.14 & 0.12 & - & -0.08 & $0.22^{* * *}$ & $0.23^{* *}$ & $0.12^{*}$ & 0.07 & 0.06 \\
\hline 7. Self-efficacy & -0.13 & -0.02 & $-0.42^{* *}$ & $-0.18^{*}$ & 0.04 & -0.05 & - & $0.25^{* *}$ & $0.14^{*}$ & -0.05 & $0.21^{* *}$ & -0.10 \\
\hline $\begin{array}{l}\text { 8. Trust in public health } \\
\text { officials }\end{array}$ & -0.06 & -0.12 & $-0.16^{*}$ & -0.10 & 0.02 & 0.07 & $0.26^{* *}$ & - & $0.64^{* * *}$ & $0.16^{* *}$ & $0.20^{* *}$ & 0.09 \\
\hline 9. Trust in doctors & 0.01 & -0.11 & 0.04 & 0.03 & 0.10 & 0.04 & 0.11 & $0.51^{* *}$ & - & 0.09 & 0.06 & 0.10 \\
\hline \multicolumn{13}{|l|}{ Moderators } \\
\hline 10. Tolerance for ambiguity & 0.10 & 0.09 & 0.09 & -0.12 & 0.07 & 0.10 & 0.00 & $0.25^{* *}$ & 0.13 & - & $0.30^{* *}$ & $0.30^{* *}$ \\
\hline 11. Dispositional optimism & -0.05 & -0.03 & -0.15 & -0.04 & 0.13 & -0.01 & $0.32^{* *}$ & $0.17^{*}$ & 0.02 & 0.00 & - & $0.37^{* *}$ \\
\hline 12. Health literacy & 0.07 & 0.07 & 0.03 & -0.07 & -0.10 & 0.06 & 0.02 & 0.04 & 0.15 & 0.04 & -0.04 & - \\
\hline Possible Range & $1-4$ & $1-4$ & $1-5$ & $1-4$ & $1-4$ & $1-4$ & $1-4$ & $1-4$ & $1-4$ & $1-4$ & $1-5$ & $1-5$ \\
\hline \multicolumn{13}{|l|}{ Experiment 1} \\
\hline M & 3.15 & 3.00 & 2.64 & 2.47 & 3.62 & 3.22 & 2.31 & 2.63 & 2.99 & 2.65 & 3.54 & 4.35 \\
\hline $\mathrm{SD}$ & 0.69 & 0.59 & 0.84 & 0.81 & 0.56 & 0.97 & 0.84 & 0.72 & 0.80 & 0.62 & 0.79 & 0.78 \\
\hline \multicolumn{13}{|l|}{ Experiment 2} \\
\hline M & 3.14 & 2.83 & 2.92 & 2.64 & 3.48 & 3.23 & 2.27 & 2.53 & 3.05 & 2.55 & 3.06 & 3.91 \\
\hline $\mathrm{SD}$ & 0.59 & 0.58 & 0.68 & 0.70 & 0.53 & 0.89 & 0.82 & 0.71 & 0.72 & 0.51 & 0.75 & 0.72 \\
\hline
\end{tabular}

* $p<0.05$

** $p<0.01$

after excluding one upper range outlier). Participants completed the study between March 30, 2020 and April 24, 2020. Data collection ended once the semester was over and we did not continue data collection in the subsequent semester despite being underpowered for a priori ANOVA analyses due to rapid changes in COVID-19 information that would have necessitate changes to our materials. One participant was excluded for not completing the majority of the survey. This exclusion was not included in the final sample size noted above. No other participants were excluded for any of the reasons specified in Experiment 1. Additionally, no participants responded "don't know" for the perceived susceptibility item.

\section{Results and discussion}

\section{Manipulation checks}

As expected (see Table 2), participants in the ambiguity condition reported significantly greater perceived message ambiguity, $M=3.35$, than participants in the control condition, $M=2.80, p<0.001$. Perceived message ambiguity did not differ among the intervention and ambiguity conditions, $p=0.425$, and those in the intervention condition, $M=3.27$, reported greater perceived message ambiguity than those in the control condition, $p<0.001$. Similar to Experiment 1, this confirmed that the message manipulation for the ambiguity condition was successful. The same pattern of effects was observed for perceived ambiguity about COVID-19 (Table 2).

\section{Differences in demographic factors and moderators by condition}

Confirming successful randomization, there were no significant differences in age, gender, race, education, tolerance for ambiguity, optimism, or health literacy (all $p$ 's $>0.268$ ) across conditions.

\section{Associations among variables}

Table 3 provides descriptive statistics of and correlations among study variables. When collapsing across conditions, perceived message ambiguity was significantly associated with greater perceived COVID-19 ambiguity. In contrast to Experiment 1, when collapsing across conditions, there were no significant associations among perceived ambiguity and the dependent variables. Specifically, individuals who reported greater perceived ambiguity about 
COVID-19 more generally reported greater perceived susceptibility to COVID-19 and greater worry about COVID19 , consistent with a pattern of pessimistic appraisal. However, perceived ambiguity about the health message was not significantly associated with any of the dependent variables, and neither index of perceived ambiguity was associated with vaccination intentions. Although the associations of perceived message ambiguity with perceived susceptibility, $r=0.16, p=0.055$, and self-efficacy, $r=-0.13, p=0.111$, did not reach statistical significance, the magnitude of the associations were similar to what was seen in Experiment 1, rs $=0.13$ and -0.16, respectively. The association of perceived message ambiguity with worry, $r=0.05, p=0.578$, was not comparable to the magnitude of the effect in Experiment 1, $r=0.18$.

\section{Main effects of condition}

As shown in Table 2, there were no main effects of condition on any of the primary or secondary dependent variables. Although not statistically significant, $p=0.230$, the pattern of severity across conditions was consistent with the main effect on severity observed in Experiment 1, such that participants in the ambiguity condition, $M=3.38$, reported lower perceived severity than participants in the control, $M=3.52$, and intervention, $M=3.55$, conditions.

\section{Moderators}

We next used hierarchical linear regressions with dummy codes to test whether condition interacted with each of three possible moderators to predict the four primary outcomes (in total, 12 regression analyses were run). There were no statistically significant interactions between condition and any of the possible moderators.

\section{Discussion}

The emergence of the global COVID-19 pandemic in the early months of 2020 has caused countless disastrous effects, including substantial death and illness (Klein et al., 2020; Slotman, 2020; UNICEF, 2020). As of late September, 2020, over 23,000 academic papers were published about COVID19 , but only 1,400 fell within the scope of health psychology (Freedland et al., 2020). Many of these were about mental disorders or stress; only a small proportion focused on health communication (4.5\%; Freedland et al., 2020). Yet, understanding what types of communications lead to engagement in preventive health behaviors-e.g., physical distancing, wearing facial coverings, and vaccination-is critical to stemming the spread of COVID-19 and future contagious diseases. Recommendations to prevent disease spread are typically behavioral and thus rely on individual action. Across two experiments, we examined the effects of an ambiguous health message about COVID-19 on beliefs underlying engagement in health behaviors and tested the efficacy of a normalization of uncertainty strategy for use within public health communications about COVID-19. Results have practical and theoretical implications.

\section{Efficacy of normalization of uncertainty interventions}

Contrary to hypotheses, participants randomly assigned to receive a normalization of uncertainty intervention-consisting of statements that it is normal and expected for scientists not to have all the information necessary at the start of an outbreak and that there is never absolute certainty when it comes to health risks-did not report lower perceived ambiguity about COVID-19 compared to participants who read unambiguous messages or ambiguous messages without the normalization intervention. The intervention also did not have a unique effect on any other health cognitions. This lack of effects regarding the intervention was demonstrated across two experimental studies functioning as direct replications of each other, with a combined sample size of 449 participants. The potential efficacy of a normalization of uncertainty intervention is supported by behavioral economic theory and empirical studies (Chow \& Sarin, 2001, 2002; Heath \& Tversky, 1991). However, the null effects from the current study replicate those of similar research conducted concerning hypothetical pandemics (Han et al., 2018; Valley et al., 2019) and revised antibiotic recommendations (Lyons et al., 2020).

The normalization of uncertainty interventions tested here and in prior research (Han et al., 2018) may have been too subtle to elicit effects. Medical professionals seem to experience naturalistic normalization of uncertainty over time: medical students became more tolerant of ambiguity the more time they spent in medical school (Han et al., 2015), and genetic counselors reported that their own uncertainty became more "routine" and expected over time (Zhong et al., 2019). Thus, normalization of uncertainty interventions might be more effective if they were longer, more detailed, and/or implemented more than once or at regular intervals. Indeed, unbeknownst to the authors of the current study, Han et al. (2021) also conducted a similar study during COVID-19 informed by Han et al. (2018). Han et al. (2021) demonstrated evidence for the efficacy of a normalization of uncertainty intervention using a longer and more detailed message. More specifically, the intervention message led to levels of perceived likelihood of getting COVID-19 and worry about COVID-19 similar to levels reported by participants in the control condition that simply emphasized uncertainty. Our normalization of uncertainty 
message was shorter than that used by Han et al. in their (2018) study because we removed portions that were not applicable to the context of COVID-19 so as not to include false information. Further, the original intervention used by Han et al. (2018) consisted primarily of direct quotes from the point of view of a healthcare professional, whereas the intervention used by Han et al. in their (2021) replication study did not consist of quotes from any particular expert. These differences across the messages might have influenced the efficacy of the various interventions. For example, individuals may be less likely to attend to information from an unknown healthcare professional. Thus, future work on normalization of uncertainty interventions should be attentive to these details of the intervention. Future work might also test and adapt other interventions aimed at reducing maladaptive responses to ambiguity, such as construal manipulations (Simonovic \& Taber, 2021) and self-affirmation (Klein et al., 2015).

\section{Effects of ambiguous versus unambiguous health information about COVID-19}

In the absence of intervention effects, we consider results concerning the main effects of ambiguity versus a control condition as well as the bivariate correlations among dependent variables (collapsed across conditions). Importantly, the ambiguity manipulation did successfully induce greater perceived ambiguity about COVID-19 compared to the control condition.

In the present study, adults randomly assigned to read an ambiguous health message about COVID-19 perceived the novel coronavirus to be less severe than participants who read an unambiguous message and those who were in the intervention condition (although this effect was only significant in Experiment 1, the pattern was the same in Experiment 2). Surprisingly, in Experiment 1, adults who reported greater perceived ambiguity about the message (regardless of condition) also reported greater perceived severity of COVID-19. This former effect provides partial support for our first hypothesis that an ambiguous message would lead to more maladaptive health cognitions and is consistent with prior research (Han et al., 2018), whereas the latter effect is consistent with theoretical predictions that greater perceived ambiguity is associated with pessimistic appraisal (Han, 2016; Viscusi, 1997; Viscusi et al., 1991). Han et al. (2018) suggest that individuals who respond to ambiguity with lower perceived severity may be engaging in motivated reasoning: the ambiguous nature of the health message may provide an opportunity to downplay disease severity (see Dieckmann et al., 2017). Indeed, the authors of one study proposed that participants engaged in motivated reasoning processes when interpreting ambiguous numerical ranges (Dieckmann et al., 2017). Further, cognitive dissonance can elicit motivated reasoning (Stone \& Wood, 2018). For example, it is possible that individuals feel cognitive dissonance from receiving conflicting information (i.e., they feel discomfort from conflicting thoughts that vaccines are effective but they are not sure whether a new COVID-19 vaccine will be effective), so they lower their perception of risk as part of motivated reasoning to resolve their cognitive dissonance and justify their avoidance behavior (i.e., "It's unlikely that I will get this illness, and if I do, it won't be that bad, so I do not have to get this vaccine"). This finding and potential interpretation has important implications for how people might behave during the pandemic, such that individuals who perceive lower severity might be more likely to engage in riskier behavior.

The associations of ambiguity with perceived susceptibility and COVID-19 worry were somewhat inconsistent: perceived susceptibility and worry did not differ across the experimental conditions, but correlations provided general support for an association of greater ambiguity with greater perceived susceptibility and worry in both studies. A recent experimental test of the effects of ambiguous information about alcohol consumption compared to a message without ambiguity found similar null effects of the ambiguity manipulation on perceived susceptibility and worry (Simonovic \& Taber, 2020), although other experimental tests have found that ambiguity leads to lower perceived susceptibility (Han et al., 2018; Pepper et al., 2019). Correlational studies have consistently shown that greater perceived ambiguity is associated with greater perceived susceptibility and worry (Han et al., 2006, 2007a, 2007b). Perhaps people who are more likely to perceive ambiguity may also be more pessimistic in other judgments, such as their beliefs about their personal disease susceptibility and worry about getting the disease. Future correlational research on perceived ambiguity may benefit from controlling for potentially confounding constructs such as neuroticism. To date, there is stronger evidence in health contexts that perceived ambiguity and pessimistic appraisals co-occur than there is to suggest that perceived ambiguity causes pessimistic appraisals.

Han et al. (2018) found that ambiguous messages about a hypothetical pandemic influenced vaccination intentions, perceived vaccine effectiveness, and perceived disease likelihood. We did not replicate these effects, which may be due to differences across the studies. One difference is that in the current study, participants may have had strong pre-existing attitudes that influenced their reaction to the manipulated health messages, but we did not measure any pre-existing beliefs. Another difference across the two studies pertains to the specific information included in the health messages. Han et al.'s (2018) ambiguity message included ambiguity about disease severity, about the probability of the disease affecting individuals, and about 
the effectiveness of the vaccine. The control message described the disease as severe and the vaccine as effective, and in both the ambiguity and control conditions, the vaccine was recommended as the best available option. In the present study, the ambiguity about the vaccine was more subtle and did not pertain to its effectiveness: both the ambiguous and control messages stated that a vaccine was in development, with the ambiguous condition further stating that "it was unknown whether or when a vaccine would be approved." The nature of main effects of ambiguity on outcomes may depend on the nature of the control or comparison condition. It is perhaps unsurprising that the control condition in Han et al.'s (2018) study -which described the disease as severe and the vaccine as effective-led to greater vaccination intentions, greater perceived vaccine effectiveness, greater perceived disease likelihood and severity, and greater trust in officials than the ambiguity condition. Future research is needed to continue untangling the effects of ambiguous information on health cognitions and intentions.

\section{Lack of moderation effects}

Contrary to hypotheses, there was little evidence that tolerance for ambiguity, optimism, or health literacy moderated how individuals responded to the health messages. Whereas null effects for tolerance for ambiguity and optimism replicate prior null effects, we did not replicate prior interactions involving health literacy (Han et al., 2018). The same scale was used in both our experiments and Han and colleagues, although Han et al. (2018) included only one of the three items in the scale. Future research is needed to continue to test the role of health literacy with respect to ambiguous health information.

\section{Limitations}

There may have been significant differences across conditions in baseline variables that were not assessed, including pre-existing beliefs and attitudes about COVID-19. Additionally, the sample for Experiment 2 was somewhat underpowered, and both studies were underpowered to identify small or small-medium effect sizes. Another limitation of the present study is that the health messages included some differences in content, such that there were more differences across the ambiguity and control conditions than is ideal in an experimental study. Our aim was to balance experimental control with capitalizing on external validity and providing accurate information about an existing pandemic; we did not wish to provide inaccurate information (1) given the sensitivity of the topic and potential for harm, and (2) to maintain ecological validity of this study with respect to an actual pandemic. Nonetheless, every attempt was made to ensure that the content of the messages was as similar as possible and only differed in the degree of ambiguity. We also ensured that the length of the messages was similar across conditions, as well as the reading level (Flesch Reading Ease: Ambiguity: 51.9, Intervention: 56.9, Control: 46.7; Flesch-Kincaid Grade Level: Ambiguity: 10.3, Intervention: 9.5, Control: 10.6). It is also possible that the title used for the control health messages ("COVID-19-What Scientists Do Know") inadvertently primed participants to think of ambiguity. However, given that we found significant differences in perceived ambiguity in the control and ambiguity conditions, it is unlikely that any such priming had a major effect on outcomes. As for the intervention group, it is possible that some participants did not read the normalization of uncertainty message considering it was placed at the end of the message, which may have contributed to the null effects that were demonstrated.

Future studies should consider including reading checks to confirm that participants attended to key information in presented messages. The current study will contribute to future reviews and meta-analyses that might assess the effectiveness of normalization of uncertainty interventions across multiple studies and contexts, as our results contribute to a body of research examining the effects of ambiguous information and interventions aimed at reducing maladaptive effects of ambiguity.

\section{Conclusion}

Determining how to communicate complex, uncertain, and potentially conflicting health information is of critical importance. Ambiguity in scientific information is not going away, and ambiguity already exists about vaccines developed for COVID-19 due to the use of novel mRNA technology (Abbasi, 2020). It is thus important to develop interventions that help individuals manage ambiguity that arises from scientific information; results suggest here that brief statements emphasizing that uncertainty is expected and normal are unlikely to be strong enough. These findings also provide additional information on how people respond to ambiguity, with important consequences: for example, a person who perceives higher ambiguity about COVID-19 and thus lower trust in health officials and doctors may then disregard medical advice.

Author's contribution NS led the study conception and design with input from JMT. Both authors contributed to data collection and statistical analysis. NS conducted the literature review and created tables. Both authors contributed to the interpretation of the findings, manuscript writing, and read and approved the final manuscript. 


\section{Declarations}

Conflicts of interest All authors declare that they have no conflicts of interest.

Ethics approval All procedures performed in studies involving human participants were in accordance with the ethical standards of the institutional and/or national research committee and with the 1964 Helsinki declaration and its later amendments or comparable ethical standards. The study was approved by Kent State University's Institutional Review Board (IRB \# 20-166).

Informed consent Informed consent was obtained from all individual participants included in the study.

Data Availability Data are available upon request from the corresponding author. Measures and messages used in this study are available on Open Science Framework (https://osf.io/n9f7x/).

\section{References}

Abbasi, J. (2020). COVID-19 and mRNA vaccines-First large test for a new approach. JAMA, 324(12), 1125-1127.

Biesecker, B. B., Klein, W. M. P., Lewis, K. L., Fisher, T. C., Wright, M. F., Biesecker, L. G., \& Han, P. K. (2014). How do research participants perceive "uncertainty" in genome sequencing? Genetics in Medicine, 16(12), 977-980.

Blaisdell, L. L., Gutheil, C., Hootsmans, N. A., \& Han, P. K. (2016). Unknown risks: Parental hesitation about vaccination. Medical Decision Making, 36(4), 479-489.

Brewer, N. T., Chapman, G. B., Gibbons, F. X., Gerrard, M., McCaul, K. D., \& Weinstein, N. D. (2007). Meta-analysis of the relationship between risk perception and health behavior: The example of vaccination. Health Psychology, 26(2), 136-145.

Camerer, C., \& Weber, M. (1992). Recent developments in modeling preferences: Uncertainty and ambiguity. Journal of Risk and Uncertainty, 5(4), 325-370.

Centers for Disease Control and Prevention. (2020, September 1). About COVID-19. https://www.cdc.gov/coronavirus/2019-ncov/ cdcresponse/about-COVID-19.html

Chew, L. D., Griffin, J. M., Partin, M. R., Noorbaloochi, S., Grill, J. P., Snyder, A., Bradley, K. A., Nugent, S. M., Baines, A. D., \& Vanryn, M. (2008). Validation of screening questions for limited health literacy in a large VA outpatient population. Journal of General Internal Medicine, 23(5), 561-566.

Chow, C. C., \& Sarin, R. K. (2001). Comparative ignorance and the Ellsberg paradox. Journal of Risk and Uncertainty, 22(2), 129-139.

Chow, C. C., \& Sarin, R. K. (2002). Known, unknown, and unknowable uncertainties. Theory and Decision, 52(2), 127-138.

de Zwart, O., Veldhuijzen, I. K., Elam, G., Aro, A. R., Abraham, T., Bishop, G. D., Voeten, H. A., Richardus, J. H., \& Brug, J. (2009). Perceived threat, risk perception, and efficacy beliefs related to SARS and other (emerging) infectious diseases: Results of an international survey. International Journal of Behavioral Medicine, 16(1), 30-40.

Dieckmann, N. F., Gregory, R., Peters, E., \& Hartman, R. (2017). Seeing what you want to see: How imprecise uncertainty ranges enhance motivated reasoning. Risk Analysis, 37(3), 471-486.

Eisenman, D. P., Williams, M. V., Glik, D., Long, A., Plough, A. L., \& Ong, M. (2012). The public health disaster trust scale: Validation of a brief measure. Journal of Public Health Management and Practice, 18(4), E11-E18.
Ellsberg, D. (1961). Risk, ambiguity, and the Savage axioms. The Quarterly Journal of Economics, 75(4), 643-669.

El-Toukhy, S. (2015). Parsing susceptibility and severity dimensions of health risk perceptions. Journal of Health Communication, 20(5), 499-511.

Faul, F., Erdfelder, E., Lang, A.-G., \& Buchner, A. (2007). G* Power 3: A flexible statistical power analysis program for the social, behavioral, and biomedical sciences. Behavior Research Methods, 39(2), 175-191.

Ferrer, R. A., \& Klein, W. M. P. (2015). Risk perceptions and health behavior. Current Opinion in Psychology, 5, 85-89.

Freedland, K. E., Dew, M. A., Sarwer, D. B., Burg, M. M., Hart, T. A., Ewing, S. W. F., Fang, C. Y., Blozis, S. A., Puterman, E., Marquez, B., \& Kaufmann, P. G. (2020). Health psychology in the time of COVID-19. Health Psychology, 39(12), 1021-1025.

Han, P. K. J. (2016). Uncertainty and ambiguity in health decisions. In M. A. Diefenbach, S. Miller-Halegoua, \& D. J., Bowen (Eds.), Handbook of health decision science (pp. 133-144). Springer.

Han, P. K., Klein, W. M. P., \& Arora, N. K. (2011a). Varieties of uncertainty in health care: A conceptual taxonomy. Medical Decision Making, 31(6), 828-838.

Han, P. K., Klein, W. M. P., Lehman, T., Killam, B., Massett, H., \& Freedman, A. N. (2011b). Communication of uncertainty regarding individualized cancer risk estimates: Effects and influential factors. Medical Decision Making, 31(2), 354-366.

Han, P. K., Kobrin, S. C., Klein, W. M. P., Davis, W. W., Stefanek, M., \& Taplin, S. H. (2007a). Perceived ambiguity about screening mammography recommendations: Association with future mammography uptake and perceptions. Cancer Epidemiology, Biomarkers \& Prevention, 16(3), 458-466.

Han, P. K., Moser, R. P., \& Klein, W. M. P. (2006). Perceived ambiguity about cancer prevention recommendations: Relationship to perceptions of cancer preventability, risk, and worry. Journal of Health Communication, 11(Suppl 1), 51-69.

Han, P. K., Moser, R. P., \& Klein, W. M. P. (2007b). Perceived ambiguity about cancer prevention recommendations: Associations with cancer-related perceptions and behaviours in a US population survey. Health Expectations, 10(4), 321-336.

Han, P. K., Reeve, B. B., Moser, R. P., \& Klein, W. M. P. (2009). Aversion to ambiguity regarding medical tests and treatments: Measurement, prevalence, and relationship to sociodemographic factors. Journal of Health Communication, 14(6), 556-572.

Han, P., Scharnetzki, E., Scherer, A. M., Thorpe, A., Lary, C., Waterston, L. B., Fagerlin, A., \& Dieckmann, N. F. (2021). Communicating scientific uncertainty about the COVID-19 pandemic: Online experimental study of an uncertainty-normalizing strategy. Journal of Medical Internet Research, 23(4), e27832.

Han, P. K., Schupack, D., Daggett, S., Holt, C. T., \& Strout, T. D. (2015). Temporal changes in tolerance of uncertainty among medical students: Insights from an exploratory study. Medical Education Online, 20, 28285.

Han, P. K., Williams, A. E., Haskins, A., Gutheil, C., Lucas, F. L., Klein, W. M. P., \& Mazor, K. M. (2014). Individual differences in aversion to ambiguity regarding medical tests and treatments: Association with cancer screening cognitions. Cancer Epidemiology, Biomarkers \& Prevention, 23(12), 2916-2923.

Han, P. K. J., Zikmund-Fisher, B. J., Duarte, C. W., Knaus, M., Black, A., Scherer, A. M., \& Fagerlin, A. (2018). Communication of scientific uncertainty about a novel pandemic health threat: Ambiguity aversion and its mechanisms. Journal of Health Communication, 23(5), 435-444.

Heath, C., \& Tversky, A. (1991). Preference and belief: Ambiguity and competence in choice under uncertainty. Journal of Risk and Uncertainty, 4(1), 5-28.

Hutchins, H. J., Wolff, B., Leeb, R., Ko, J. Y., Odom, E., Willey, J., Friedman, A., \& Bitsko, R. H. (2020). COVID-19 mitigation 
behaviors by age group-United States, April-June 2020. Morbidity and Mortality Weekly Report, 69(43), 1584.

Jensen, J. D., Pokharel, M., Scherr, C. L., King, A. J., Brown, N., \& Jones, C. (2017). Communicating uncertain science to the public: How amount and source of uncertainty impact fatalism, backlash, and overload. Risk Analysis, 37(1), 40-51.

Katz, S. J., Erkkinen, M., Lindgren, B., \& Hatsukami, D. (2018). Assessing the impact of conflicting health warning information on intentions to use e-cigarettes-an application of the HeuristicSystematic model. Journal of Health Communication, 23(10-11), 874-885.

Katz, S. J., Lindgren, B., \& Hatsukami, D. (2017). E-cigarettes warning labels and modified risk statements: Tests of messages to reduce recreational use. Tobacco Regulatory Science, 3(4), 445-458.

Klein, P. (Producer), Brandt, C. (Producer), Linderman., J. (Producer), Mendoza, M. (Producer), \& McCormick, K. (Producer). (2020). America's medical supply crisis [Film]. Frontline PBS.

Klein, W. M. P., \& Ferrer, R. A. (2018). On being more amenable to threatening risk messages concerning close others (vis-à-vis the self). Personality and Social Psychology Bulletin, 44(10), 1411-1423.

Klein, W. M. P., Lipkus, I. M., Scholl, S. M., McQueen, A., Cerully, J. L., \& Harris, P. R. (2010). Self-affirmation moderates effects of unrealistic optimism and pessimism on reactions to tailored risk feedback. Psychology and Health, 25(10), 1195-1208.

Liang, M., Gao, L., Cheng, C., Zhou, Q., Uy, J. P., Heiner, K., \& Sun, C. (2020). Efficacy of face mask in preventing respiratory virus transmission: A systematic review and meta-analysis. Travel Medicine and Infectious Disease, 36, 101751.

Lipkus, I. M., Green, L. G., \& Marcus, A. (2003). Manipulating perceptions of colorectal cancer threat: Implications for screening intentions and behaviors. Journal of Health Communication, 8(3), 213-228.

Lyons, B. A., Merola, V., \& Reifler, J. (2020). Shifting medical guidelines: Compliance and spillover effects for revised antibiotic recommendations. Social Science \& Medicine, 255, 112943.

Meszaros, J. R., Asch, D. A., Baron, J., Hershey, J. C., Kunreuther, H., \& Schwartz-Buzaglo, J. (1996). Cognitive processes and the decisions of some parents to forego pertussis vaccination for their children. Journal of Clinical Epidemiology, 49(6), 697-703.

Moreland, A., Herlihy, C., Tynan, M. A., Sunshine, G., McCord, R. F., Hilton, C., Poovey, J., Werner, A. K., Jones, C. D., Fulmer, E. B., Gundlapalli, A. V., Strosnider, H., Potvien, A., García, M. C., Honeycutt, S., \& Baldwin, G. (2020). Timing of state and territorial COVID-19 stay-at-home orders and changes in population movement-United States, March 1-May 31 2020. Morbidity and Mortality Weekly Report, 69, 1198-1203.

Pepper, J. K., Squiers, L. B., Peinado, S. C., Bann, C. M., Dolina, S. D., Lynch, M. M., Nonnemaker, J. M., \& McCormack, L. A. (2019). Impact of messages about scientific uncertainty on risk perceptions and intentions to use electronic vaping products. Addictive Behaviors, 91, 136-140.

Ritov, I., \& Baron, J. (1990). Reluctance to vaccinate: Omission bias and ambiguity. Journal of Behavioral Decision Making, 3(4), 263-277.

Schapira, M. M., Aggarwal, C., Akers, S., Aysola, J., Imbert, D., Langer, C., Simone, C. B., II., Strittmatter, E., Vachani, A., \& Fraenkel, L. (2016). How patients view lung cancer screening. The role of uncertainty in medical decision making. Annals of the American Thoracic Society, 13(11), 1969-1976.

Scheier, M. F., Carver, C. S., \& Bridges, M. W. (1994). Distinguishing optimism from neuroticism (and trait anxiety, self-mastery, and self-esteem): A reevaluation of the Life Orientation Test. Journal of Personality and Social Psychology, 67(6), 1063.

Simonovic, N., \& Taber, J. M. (2020). Perceptions of ambiguity about cigarettes and e-cigarettes among users and non-users. Society of
Behavioral Medicine 2020 Annual Meeting Abstracts. Annals of Behavioral Medicine, 54(Suppl 1), S665-S665.

Simonovic, N., \& Taber, J. M. (2021). Effects of construal level on responses to ambiguous health information about alcohol consumption. Health Communication, 2, 1-14.

Simonovic, N., Taber, J. M., Klein, W. M. P., \& Ferrer, R. A. (2020). Evidence that perceiving and being averse to medical ambiguity are distinct constructs: An analysis of nationally representative U.S. data. Health Expectations, 23(3), 603-613.

Slotman, J. R. (2020, October 15). UN/DESA Policy Brief \#86: The long-term impact of COVID-19 on poverty. https://www.un.org/ development/desa/dpad/publication/un-desa-policy-brief-86-thelong-term-impact-of-covid-19-on-poverty/

Stone, D. F., \& Wood, D. H., (2018). Cognitive dissonance, motivated reasoning, and confirmation bias: Applications in industrial organization. In V. J. Tremblay, E. Schroeder, \& C. H. Tremblay (Eds.), Handbook of behavioral industrial organization. Edward Elgar Publishing.

Taber, J. M., Klein, W. M. P., Ferrer, R. A., Han, P. K., Lewis, K. L., Biesecker, L. G., \& Biesecker, B. B. (2015). Perceived ambiguity as a barrier to intentions to learn genome sequencing results. Journal of Behavioral Medicine, 38(5), 715-726.

Taber, J. M., McQueen, A., Simonovic, N., \& Waters, E. A. (2019). Adapting a self-affirmation intervention for use in a mobile application for smokers. Journal of Behavioral Medicine, 42(6), $1050-1061$.

UNICEF (2020, August). Covid-19: Are children able to continue learning during school closures? A global analysis of the potential reach of remote learning policies using data from 100 countries. https://data.unicef.org/resources/remote-learning-reachabili ty-factsheet/

Valley, T., Scherer, A., Knaus, M., Zikmund-Fisher, B., Das, E., \& Fagerlin, A. (2019). Prior vaccination and effectiveness of communication strategies used to describe infectious diseases. Emerging Infectious Disease, 25(4), 821.

Viscusi, W. K. (1997). Alarmist decisions with divergent risk information. The Economic Journal, 107(445), 1657-1670.

Viscusi, W. K., Magat, W. A., \& Huber, J. (1991). Communication of ambiguous risk information. Theory and Decision, 31(2-3), 159-173.

Weinstein, N. D., Kwitel, A., McCaul, K. D., Magnan, R. E., Gerrard, M., \& Gibbons, F. X. (2007). Risk perceptions: Assessment and relationship to influenza vaccination. Health Psychology, 26(2), 146-151.

World Health Organization (2020a, September 9). Timeline of WHO's response to COVID-19. https://www.who.int/news/item/29-062020-covidtimeline

World Health Organization (2020b, October 26). WHO Director-General's opening remarks at the media briefing on COVID-19-26 October 2020. https://www.who.int/news/item/29-06-2020-covid timeline

Yang, H., Varley-Campbell, J., Coelho, H., Long, L., Robinson, S., Snowsill, T., Griffin, E., Peters, J., \& Hyde, C. (2019). Do we know enough about the effect of low-dose computed tomography screening for lung cancer on survival to act? A systematic review, meta-analysis and network meta-analysis of randomised controlled trials. Diagnostic and Prognostic Research, 3(1), 1-10.

Zhong, L., Woo, J., Steinhardt, M. A., \& Vangelisti, A. L. (2019). "Our job is that whole gray zone in between there": Investigating genetic counselors' strategies for managing and communicating uncertainty. Health Communication, 35(13), 1-10.

Publisher's Note Springer Nature remains neutral with regard to jurisdictional claims in published maps and institutional affiliations. 